\title{
Heart Rate Variability in an Elite Female Alpine Skier: a Case Study
}

\author{
${ }^{1}$ Sean Wallace, ${ }^{2}$ Matt Jordan, ${ }^{1}$ Tracy Blake, ${ }^{1}$ Patricia Doyle-Baker ${ }^{*}$ \\ ${ }^{1}$ Faculty of Kinesiology, University of Calgary, Calgary, Alberta, Canada. ${ }^{2}$ Cumming School of Medicine, Department of \\ Medical Sciences, University of Calgary, Calgary, Alberta, Canada.
}

\begin{abstract}
Objectives. The purpose of this study was to assess the heart rate variability (HRV) during off-snow and on-snow training in an elite female alpine ski racer. Methods. Using a case study design, a single elite female alpine ski racer ( 25 years old; $175.6 \mathrm{~cm} ; 69.9 \mathrm{~kg}$ ) was recruited from the Canadian Alpine Ski Team. Training load was obtained using the sessional rating of perceived exertion method (sRPE), and a weekly sum was calculated using all training loads in a calendar week. Resting heart rate was recorded upon waking using a heart rate monitor. HRV was calculated using the natural logarithms of the root mean square of the successive differences of R-R intervals (lnRMSSD), and the coefficient of variation of lnRMSSD (lnRMSSDCV) with smallest worthwhile change (SWC). Compliance was $19.53 \%$. Results. An inverse relationship was identified between the extreme values for lnRMSSD and SRPE. Daily lnRMSSDCV found two time-points that were significantly greater than SWC. The regression analysis of daily lnRMSSDCV over time had a positive slope of $0.001(\mathrm{R}=0.0029)$. Three major depressions in lnRMSSD were observed over the recording period and two coincided with peak sRPE. The largest depression occurred on the same day the subject sustained a shoulder dislocation during a routine strength training session. The subject maintained training status over the training period but it was not predictive of future performance. Conclusion. In this case study, the low compliance limited the ability to identify possible relationships; however periods of functional overreach were identifiable.
\end{abstract}

KEY WORDS: Alpine ski, HRV, Training, Variability, Overreach.

\section{INTRODUCTION}

To maximize performance, elite athletes perform highly structured, periodized training regimens in which training loads and recovery periods are systematically manipulated to elicit peak performance (1). These programs contain periods of functional overreaching designed to disturb homeostasis and prompt adaptations that result in improved performance (2). If an athlete does not recover or is unable to adapt to the stimuli, they enter a state of nonfunctional overreaching (1). Nonfunctional overreaching is characterized by a period of stagnation or performance decrease that may last for several weeks (1). If this period of maladaptation is prolonged, overtraining can occur, which is characterized by significant fatigue, sustained performance decline, and hormonal changes (1, 3). To avoid overtraining, it is essential that coaches detect nonfunctional overreaching. However, it is difficult to objectively distinguish between these three different states (2).

*. Corresponding Author:

Patricia Doyle-Baker

E-mail: pdoyleba@ucalgary.ca 
To improve the detection of nonfunctional overreaching and overtraining, objective measures of an athlete's readiness to train have become increasingly sought-after (1). Autonomic dysfunction has been proposed as part of the pathogenesis of overtraining in endurance athletes (4) the assessment of heart rate variability (HRV) has been proposed as a suitable metric for detecting changes in autonomic regulation that can indicate a transition from a state of functional overreaching to nonfunctional overreaching.

HRV refers to the natural oscillations in the timing of consecutive heartbeats as defined by the 1996 Task Force of the European Society of Cardiology and the North American Society for Pacing and Electrophysiology (5) and is controlled by the autonomic nervous system through the antagonistic actions of the sympathetic and parasympathetic system (6). An increase in HRV has been associated with a positive change in fitness scores, the ability to tolerate higher levels of exercise intensity and cardioprotective effects (7) and a decrease in HRV may be associated with nonfunctional overreached states (1).

Longitudinal monitoring of HRV by calculating the natural logarithm of the root mean square of the successive differences of the $\mathrm{R}-\mathrm{R}$ intervals (InRMSSD) derived from daily short-term measurements has been recommended for use with athletes who train more than four days per week to evaluate readiness for training and competition $(8,9)$ Additionally, the smallest worthwhile change in HRV can be established in longitudinal settings by calculating a coefficient of variation for the lnRMSSD $\left(\operatorname{lnRMSSD}_{\mathrm{CV}}\right)$ over repeated measurements in an individual. This approach was recently used to map the transition into a nonfunctional overreached state in a case study of two male national level triathletes (9). In this case, a marked decrease in the $\operatorname{lnRMSSD}_{\mathrm{CV}}$ for one of the athletes was observed in the weeks prior to competition, which according to Plews et al. (2012) indicated a transition into a nonfunctional overreached state and consequently poor competition performance (9).

The majority of the studies evaluating the efficacy of HRV measurements in athlete populations have focused on male endurance athletes. However, the potential for maladaptation to training and fatigue are important for technically demanding sports such as elite alpine ski racing due to the high risk for serious injuries (10). These injuries often occur in the final sections of races presumably when fatigue factors are greater contributors to the injury event (11). Additionally, elite alpine ski racers participate in rigorous off-snow physical training programs and display high levels of physical fitness $(12,13)$. Fitness level is perceived as important modifiable factor for the prevention of injuries amongst athletes and coaches (14). Given that physical fitness and the importance of monitoring fatigue for injury prevention strategies, investigating the utility of objectively determined metrics such as HRV may be extremely useful to sports science practitioners training elite athletes. However, to the best of our knowledge there are no published studies on the efficacy of longitudinal HRV monitoring in elite female alpine ski racing populations.

The primary purpose of this study was to investigate the feasibility and application of longitudinal HRV monitoring in an elite female alpine ski racer in a real-world, highperformance training environment. A secondary objective was to investigate whether HRV changes would be predictive of performance and adaptation to training load.

\section{MATERIALS AND METHODS}

Participants. One elite female alpine ski racer (25 years old; $175.6 \mathrm{~cm} ; 69.9 \mathrm{~kg}$ ) on the Canadian Alpine Ski Team was recruited. This subject competed at the World Cup, World Championship, and Olympic level in all disciplines (slalom, giant slalom, super G, super combined and downhill). Informed consent was obtained from the subject and ethical approval was received by the Conjoint Health Research Ethics Board at the University of Calgary, (Alberta, Canada) in early 2015.

Study Duration. The study period was May 19, 2014 to January 31, 2015 (257 days). A week was defined corresponding to the calendar year of Sunday to Saturday. The study period included the athlete's off-snow physical fitness training, on-snow training and part of her 
competition season, which began on November 15, 2015 (Day 180).

Training Load. Physical training load was defined as the dose of training completed by the athlete during an exercise bout (15). To quantify training load, the sessional rating of perceived effort (sRPE) method was used, which is the product of the RPE and training duration in minutes (16). This is a validated approach for assessing training load in athlete populations (15). The method requires the athlete to assess RPE for the entire training session using the category ratio scale (CR-10) (17). The athlete entered her RPE and the training time in minutes using a secure online questionnaire within 30 minutes of completing a training session. For days with multiple training sessions, the sRPE for each individual training session was calculated and a daily training load was computed as the sum of the sRPE for all the training sessions on that specific day.

Heart Rate Variability. HRV was collected via $\mathrm{R}-\mathrm{R}$ intervals measured by a Polar RS800cx heart monitor (RS800cx; Polar Electro, Kemple, Finland). The R-R intervals were recorded immediately upon the athlete waking from sleep. The athlete was instructed to leave the heart monitor beside her bed before going to sleep, to minimize disturbance when putting on the heart monitor. The athlete was instructed to lie in a supine position after the heart monitor had been fitted securely around her chest. The recordings were six minutes in duration, synchronized and saved to the athlete's mobile phone. The recordings were later uploaded to a secure laptop. The R-R series intervals were analyzed individually using a custom Excel software program by first excluding artifacts and ectopic heart beats. All tachograms were visually inspected to ensure that irregular beats were not a natural phenomenon. The root mean square of the successive differences (RMSSD) was calculated from the middle segment of each recording, corresponding to 150 to 450 seconds ( 5 minutes) of the recording, as recommended by the 1996 Task Force of the European Society of Cardiology and the North American Society for Pacing and Electrophysiology (5). Daily and weekly RMSSD values were calculated. Weekly RMSSD was calculated by taking the mean of all the RMSSD measures for that week. The athlete's compliance for taking the morning HRV was calculated as the total number of days of morning HRV recordings over the total number of days in the study period.

Data Analysis. Due to the skewed nature of HRV measures, the RMSSD values were log transformed using the natural logarithm (ln) (18). The coefficient of variation was calculated for daily $\operatorname{lnRMSSD}\left(\operatorname{lnRMSSD}_{\mathrm{CV}}\right)$. LnRMSSD $_{\mathrm{CV}}$ was calculated as a percentage and the smallest worthwhile change was calculated as a difference of $\pm 0.5 \%$ from the mean $\operatorname{lnRMSSD} \mathrm{CV}_{\%}$ as recommended (19). Linear regression was used on daily $\operatorname{lnRMSSD}_{\mathrm{Cv}}$ values leading up to the athlete's competition season (day 180) to predict improvement or declination in performance. The magnitude of the relationship between time and $\operatorname{lnRMSSD}_{\mathrm{CV}}$ was assessed using the modified criteria of Cohen (20).

\section{RESULTS}

Over the study period, the athlete's mean hours of sleep per night was $( \pm \mathrm{SD}$; range) 8.8 \pm 1.3 (5-14), and mean RPE was $3.4 \pm 1.3$ (1-8). The athlete provided HRV recordings on $19.53 \%$ of total days (257 days). Compliance had a general negative trend with a sharp drop at the beginning of competition period as presented in Figure 1. The compliance rate was dynamic, varying from $3 \%$ to $37 \%$ over the study period. In general, greater compliance was observed in the off-snow physical training period and lower compliance occurred during the competition season.

The weekly lnRMSSD and weekly sum of the training load recorded over the study period are presented in Figure 2. The weekly lnRMSSD had a mean $( \pm$ SD) of $4.70( \pm 0.40)$, with three noticeable depressions at weeks 8,17 and 33 . The mean $( \pm \mathrm{SD})$ weekly training load over the study period was $3744( \pm 1807)$. Peak weekly training load occurred at week 8 (7678) and week 33 (7650), which coincided with the depressions in weekly lnRMSSD of 4.27 and 4.30 in weeks 8 and 33, respectively.

The two lowest weekly training load occurred at Week 6 (1080) and Week 20 (1080), and did not appear to coincide with the weekly $\operatorname{lnRMSSD}$. 
The largest depression in weekly lnRMSSD occurred during Week 17 (lnRMSSD=3.32), which were three standard deviations below the mean. This measurement was the only HRV recording taken during this week, and did not coincide with a significant increase in the training load. However, at the morning training session on the same day that the HRV recording was taken, the athlete suffered an injury (shoulder dislocation) during a routine strength training exercise.

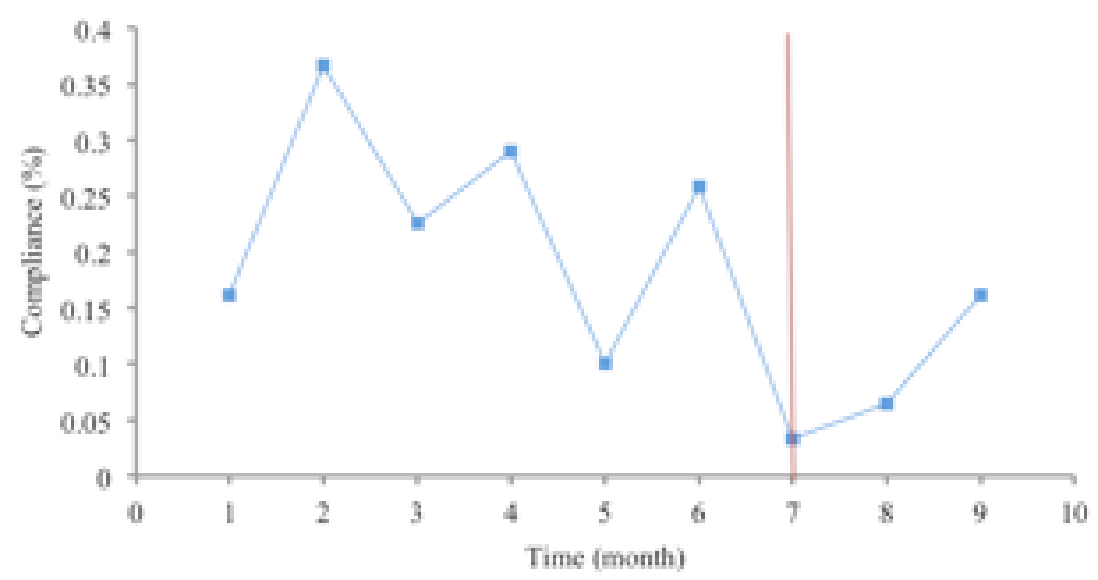

Figure 1. Athlete's monthly compliance for recording morning HRV measures over the nine month study period. Superimposed line indicates separation of training period (left) and competition period (right), which started on the November 15, 2014.

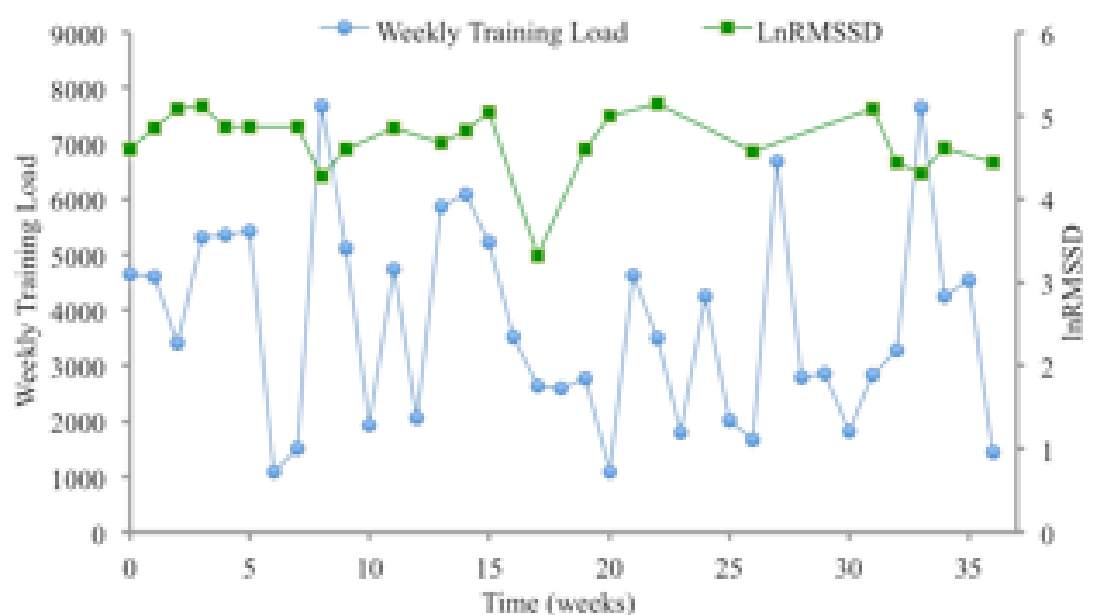

Figure 2. Weekly average $\operatorname{lnRMSSD}($ mean $=4.695 \pm 0.397)$ and weekly training load $($ mean $=3744.5 \pm 1807.7)$ recorded over entire 36 week study period.

The results for daily $\operatorname{lnRMSSD}_{\mathrm{CV}}$ over the study period are presented in Figure 3. Daily $\operatorname{lnRMSSD}_{\mathrm{CV}}$ had a mean value of $9.56 \%$ and the smallest worthwhile change was calculated with an upper bound of $10.06 \%$ and a lower bound of $9.06 \%$. There were two outliers; at Day 56 (daily
$\operatorname{lnRMSSD} D_{\mathrm{CV}}=12.11$ ), and at day 122 (daily $\operatorname{lnRMSSD} \mathrm{CV}_{\mathrm{CV}}=13.56 \%$ ).

Regression analysis performed on the daily $\operatorname{lnRMSSD} \mathrm{CV}_{\mathrm{C}}$ for the 17 weeks leading up to the competition period found a positive general trend of $0.001 \%$ per day over the training period. A small magnitude relationship existed between 
time and $\operatorname{lnRMSSD} \mathrm{D}_{\mathrm{CV}}(\mathrm{R}=0.0029)$. The athlete had underperformed during the competition

period relative to her past performance and these results are presented in Figure 4.

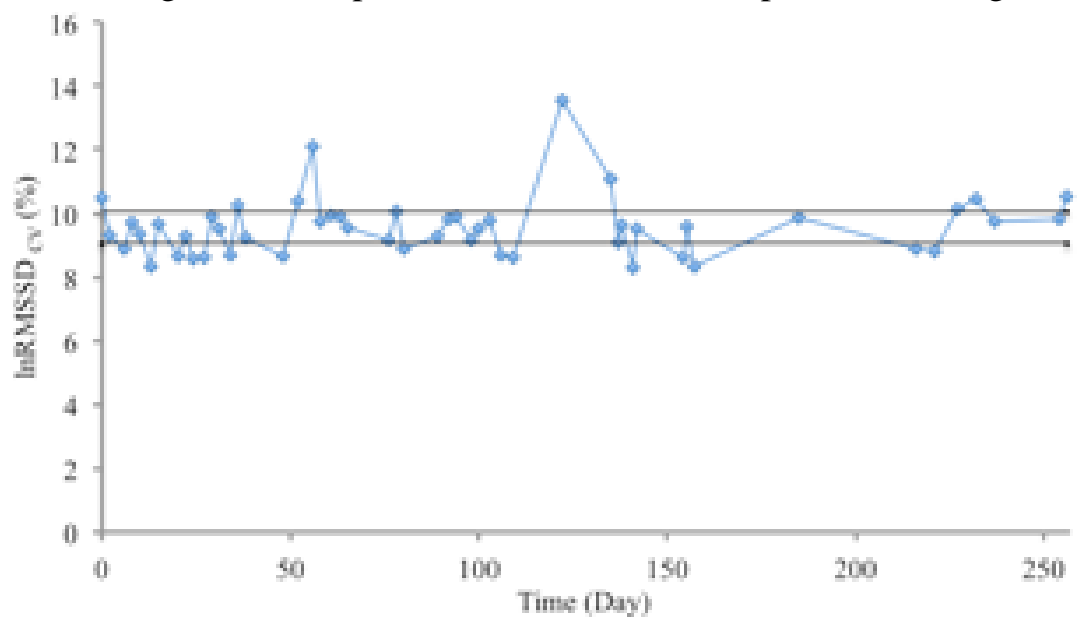

Figure 3. Coefficient of variation for daily lnRMSSD calculated over entire study period. Smallest worthwhile change upper and lower bound are superimposed onto the figure as horizantal black lines.

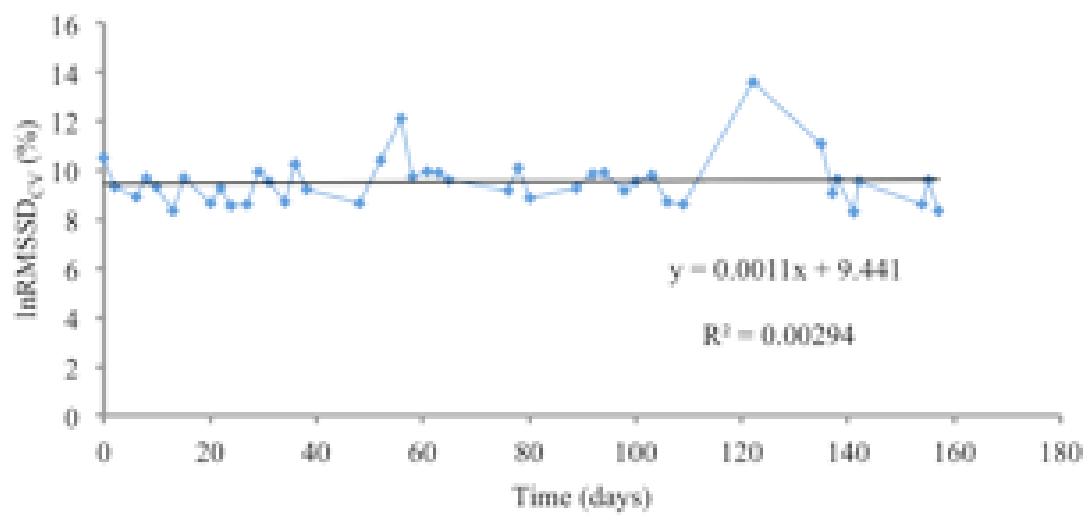

Figure 4. Regression analysis of daily $\operatorname{lnRMSSD}_{\mathrm{CV}}$ during training period leading up to competition period (day 180).

\section{DISCUSSION}

The primary purpose of our study was to investigate the feasibility and application of HRV monitoring to assess training readiness in an elite female alpine ski racer. The secondary purpose was to evaluate the predictive value of HRV for performance. The subject had a compliance of $19.53 \%$ during the study period, with a noticeable difference between the training period and the competition period. Previous research has recommended a compliance of three to four recordings per week for accurate assessment of athlete's readiness using HRV measurements (8). We conducted our study over a typical season including off-snow physical training period and in-season competition period to address the efficacy of HRV monitoring in elite alpine ski racers. Based on our finding, it appears there is specific need to address the challenges of obtaining HRV recordings throughout the competitive season. Emphasizing the importance of maintaining compliance during all periods of the season by coaches may help mitigate this problem (21).

The weekly lnRMSSD was stable over the course of the study period, with the exception of three noticeable depressions. Two of the depressions in weekly lnRMSSD corresponded with peak training load values reported during the same week (Week 8 and Week 33). HRV 
depression following high intensity training occurs as a result of increased sympathetic modulation of the heart rate (22). Our results suggest a similar utility for HRV monitoring in our athlete. The subsequent recovery of HRV to normal levels after depression would indicate that the athlete had adequately adapted to the training load (8).

The combination of altitude and the high daily training load may explain the decrease in lnRMSSD recorded during week 8 . During week 8 , the athlete was training at altitude, which has previously been shown to decrease HRV indices, as a result of increased sympathetic activity (23).

The largest depression in the weekly lnRMSSD occurred during Week 17 and did not coincide with an elevated training load. However, at the morning training session after the HRV recording was obtained, the athlete suffered a shoulder dislocation during a routine strength training exercise. Due to the challenges in obtaining consistent HRV recordings during specific weeks of the study period, only a single HRV measurement was made during this week. To our knowledge no study has previously been published regarding decreased HRV and possible risk of injury. Further research is warranted to investigate this possibility given the high risk for serious injury in elite alpine racing (10). The large drop in HRV may indicate autonomic disturbance, but the source of the disturbance remains unknown. Other research has shown that decreased HRV is indicative of negative health consequences, such as disease states (5).

The minimal weekly training load values, week 6 (1080) and week 20 (1080), did not have a noticeable effect on weekly lnRMSSD. Previous research has shown there is a reduced autonomic response to low intensity exercise (23). It is possible that training intensities during these weeks were too low to elicit a noticeable autonomic response during longitudinal monitoring.

The majority of daily $\operatorname{lnRMSSD}_{\mathrm{CV}}$ data points fell within or just outside the range for the smallest worthwhile change. The same time points that were noticeably lower than the lower bound of smallest worthwhile change corresponded with Week 8 and Week 17 of the weekly lnRMSSD, which had a depressed HRV.
As previously mentioned, these time periods corresponded with heavy training, including being at altitude and a measurement taken prior to a serious injury.

Regression analysis of daily $\operatorname{lnRMSSD}_{\mathrm{CV}}$ leading up to competition period showed an improvement of $0.001 \%$ per day over the training period. Previous research into the predictive value of HRV demonstrate improvements of 0.3 to 0.4 of the individual CV will predict improved performance (19). Our athlete had a subtle trend, which would be indicative of maintained performance. Additionally, in comparison to the previous season, our subject demonstrated a slight drop in competitive performance. Thus, regression analysis of CV may only be indicative of impaired performance in the presence of nonfunctional overreaching or overtraining as previously reported (9).

\section{CONCLUSION}

Despite less than consistent reporting, HRV was sensitive enough to detect changes in an elite female alpine ski racer's training status, specifically when the athlete entered phases of functional overreaching and subsequent recovery. Unlike previous studies (9), regression analysis of $\operatorname{lnRMSSD}_{\mathrm{CV}}$ was not predictive of future performance. This result may represent a difference in the utility of HRV in predicting performance for alpine ski racing compared to endurance sports. Research in this area is limited and warrants further investigation into the expected HRV responses for athletes competing in extreme and technically demanding sports versus endurance athletes. There was a potential for a publication bias due to the limited research using HRV in elite athletes, and therefore more research is necessary to discern the true relationship between HRV and training in elite athletes. HRV may represent a feasible and valid tool for assessing training status in elite female alpine skiers. Future research should focus on the potential value for using longitudinal HRV monitoring to predict performance, identify maladaptation to training and injury risk in a larger group of subjects particularly females, with specific measures to mitigate low compliance during in season competition periods. 
We were limited to presenting anomalies that were specific to one individual, thus may not be generalizable to other elite female alpine ski racers. It is challenging to study elite athletes in real world training and competition environments, however, it is important to conduct research specific to their highly specialized characteristics and circumstances. The poor compliance obtained in specific periods of this study limited our ability to fully interpret the HRV data. Methods for improving compliance will need to be implemented for future research wishing to use HRV to be an objective tool to assess training status.

\section{ACKNOWLEDGEMENTS}

This research was conducted jointly through the Canadian Sports Institute and the Faculty of Kinesiology at the University of Calgary in Alberta Canada. Funding support was through the Sports Science Association of Alberta through Alberta Sport Connection. The author's have no conflicts of interest that are directly relevant to the content of this article.

\section{APPLICABLE REMARKS}

- The majority of the studies evaluating the efficacy of HRV measurements in athlete populations have focused on male endurance athletes.

- This pilot study has identified that HRV can be sensitive enough to detect changes in elite female alpine racer's training status, specifically in the areas functional overreach and recovery.

\section{REFERENCES}

1. Meeusen R, Duclos M, Foster C, Fry A, Gleeson M, Nieman D, et al. Prevention, diagnosis, and treatment of the overtraining syndrome: joint consensus statement of the European College of Sport Science and the American College of Sports Medicine. Medicine and science in sports and exercise. 2013;45(1):186-205.

2. Schmitt L, Regnard J, Desmarets M, Mauny F, Mourot L, Fouillot JP, et al. Fatigue shifts and scatters heart rate variability in elite endurance athletes. PloS one. 2013;8(8):e71588.

3. Foster C. Monitoring training in athletes with reference to overtraining syndrome. Medicine and science in sports and exercise. 1998;30(7):1164-8.

4. Kiviniemi AM, Tulppo MP, Hautala AJ, Vanninen E, Uusitalo AL. Altered relationship between R-R interval and R-R interval variability in endurance athletes with overtraining syndrome. Scandinavian journal of medicine \& science in sports. 2014;24(2):e77-85.

5. Heart rate variability: standards of measurement, physiological interpretation and clinical use. Task Force of the European Society of Cardiology and the North American Society of Pacing and Electrophysiology. Circulation. 1996;93(5):1043-65.

6. Aubert AE, Seps B, Beckers F. Heart rate variability in athletes. Sports medicine (Auckland, NZ). 2003;33(12):889-919.

7. Furholz M, Radtke T, Roten L, Tanner H, Wilhelm I, Schmid JP, et al. Training-related modulations of the autonomic nervous system in endurance athletes: is female gender cardioprotective? European journal of applied physiology. 2013;113(3):631-40.

8. Buchheit M. Monitoring training status with HR measures: do all roads lead to Rome? Frontiers in physiology. 2014;5:73.

9. Plews DJ, Laursen PB, Kilding AE, Buchheit M. Heart rate variability in elite triathletes, is variation in variability the key to effective training? A case comparison. European journal of applied physiology. 2012;112(11):3729-41.

10. Flørenes TW, Bere T, Nordsletten L, Heir S, Bahr R. Injuries among male and female World Cup alpine skiers. British Journal of Sports Medicine. 2009;43(13):973-8.

11. Bere T, Florenes TW, Krosshaug T, Haugen P, Svandal I, Nordsletten L, et al. A systematic video analysis of 69 injury cases in World Cup alpine skiing. Scandinavian journal of medicine \& science in sports. 2014;24(4):66777.

12. Jordan MJ, Aagaard P, Herzog W. Rapid hamstrings/quadriceps strength in ACL-reconstructed elite Alpine ski racers. Medicine and science in sports and exercise. 2015;47(1):109-19.

13. Neumayr G, Hoertnagl H, Pfister R, Koller A, Eibl G, Raas E. Physical and physiological factors associated with success in professional alpine skiing. International journal of sports medicine. 2003;24(8):571-5. 
14. Spörri J, Kröll J, Amesberger G, Blake OM, Müller E. Perceived key injury risk factors in World Cup alpine ski racing - an explorative qualitative study with expert stakeholders. British Journal of Sports Medicine. 2012;46(15):1059-64.

15. Wallace LK, Slattery KM, Coutts AJ. A comparison of methods for quantifying training load: relationships between modelled and actual training responses. European journal of applied physiology. 2014;114(1):11-20.

16. Foster C, Hector LL, Welsh R, Schrager M, Green MA, Snyder AC. Effects of specific versus cross-training on running performance. European journal of applied physiology and occupational physiology. 1995;70(4):367-72.

17. Borg G, Hassmen P, Lagerstrom M. Perceived exertion related to heart rate and blood lactate during arm and leg exercise. European journal of applied physiology and occupational physiology. 1987;56(6):679-85.

18. Nunan D, Sandercock GR, Brodie DA. A quantitative systematic review of normal values for short-term heart rate variability in healthy adults. Pacing and clinical electrophysiology : PACE. 2010;33(11):1407-17.

19. Hopkins WG, Hawley JA, Burke LM. Design and analysis of research on sport performance enhancement. Medicine and science in sports and exercise. 1999;31(3):472-85.

20. Cohen J. Statistical Power Analysis for the Behavioral Sciences: Taylor \& Francis; 2013. 567 p.

21. Kovacs MS, Pritchett R, Wickwire PJ, Green JM, Bishop P. Physical performance changes after unsupervised training during the autumn/spring semester break in competitive tennis players. British Journal of Sports Medicine. 2007;41(11):705-10.

22. Iellamo F, Legramante JM, Pigozzi F, Spataro A, Norbiato G, Lucini D, et al. Conversion from vagal to sympathetic predominance with strenuous training in high-performance world class athletes. Circulation. 2002;105(23):2719-24.

23. Casonatto J, Tinucci T, Dourado AC, Polito M. Cardiovascular and autonomic responses after exercise sessions with different intensities and durations. Clinics. 2011;66(3):453-8. 